\title{
Hospital-Based Management of Diabetes Mellitus
}

\author{
William T. Ford Jr. • Gerard X. Brogan Jr. • \\ Gregory Volturo $\cdot$ Sameer Badlani $\cdot$ \\ Charles V. Pollack Jr. • David J. Yu
}

Published online: 14 January 2014

(C) Springer Science+Business Media New York 2014

\begin{abstract}
Patients with insulin-dependent diabetes mellitus or noninsulin-dependent diabetes mellitus are frequently admitted to a hospital, usually for primary conditions other than diabetes. In addition to endorsing tight glycemic control in critical care units, current standards of care recommend treatment targets similar to those of outpatients for noncritically ill patients in hospital. Given the current prevalence of diabetes (DM), how best to address hyperglycemia in the Emergency Department (ED) is an increasingly relevant issue in which the principal goals are to minimize disruption of the metabolic state and achieve a stable glycemic balance as promptly as possible. Transition of care from the ED to an inpatient service can result in gaps in comprehensive care, particularly in patients with DM who may have other serious comorbidities. In critically ill patients, insulin therapy should be initiated for treatment of persistent hyperglycemia, starting at $>180 \mathrm{mg} / \mathrm{dL}$ (10 mmol/ L). After insulin therapy has been started, the standard of care recommends a random glucose range of $140-180 \mathrm{mg} / \mathrm{dL}$ $(7.8-10 \mathrm{mmol} / \mathrm{L})$ for the majority of hospitalized patients. The traditional model of care among hospital-based physicians has been to treat the diabetic patient with a regular insulin on a sliding scale. Current evidence suggests that while this
\end{abstract}

W. T. Ford Jr.

Temple University School of Medicine, Philadelphia, PA, USA

W. T. Ford Jr.

Abington Health, Abington, PA, USA

G. X. Brogan Jr. · G. Volturo · S. Badlani ·

C. V. Pollack Jr. · D. J. Yu ( $₫)$

Department of Emergency Medicine, Hofstra North Shore LIJ

School of Medicine, Hempstead, NY, USA

e-mail: davidyumd@gmail.com

G. X. Brogan Jr.

e-mail: GBrogan@NSHS.edu approach is straightforward, it may not be the best for patient care. Patients who present to the ED or are hospitalized often require an insulin regimen that can change rapidly as their condition changes. A basal/bolus insulin regimen fulfills those criteria and mimics a normal insulin profile.

Keywords Diabetes related heart disease · Insulindependent diabetes mellitus - Emergency and hospital medicine Cardiovascular health · Noninsulin-dependent diabetes mellitus - Hospital-based management of DM

\section{Introduction}

Patients with insulin-dependent diabetes mellitus (IDDM) or noninsulin-dependent diabetes mellitus (NIDDM) are frequently admitted to a hospital, usually for primary conditions other than diabetes. Diabetics are more likely to be hospitalized and have a longer length of hospital stay (LOS) compared with those without diabetes. Substantial observational evidence links hyperglycemia to poor outcomes in hospitalized patients. The American Diabetes Association (ADA) has therefore added recommendations for treatment of hyperglycemia in hospitalized patients to its annual Standards of Medical Care [ $1 \bullet]$.

In addition to endorsing tight glycemic control in critical care units, these standards of care recommend treatment targets similar to those of outpatients for noncritically ill patients in hospital. The latter recommendation is based on a lack of randomized controlled trial (RCT) evidence for treatment targets and glycemic goals in this population. The efforts of the ADA and the American Association of Clinical Endocrinologists (AACE) have contributed to a growing national movement in which the management of inpatient hyperglycemia is seen as a quality-of-care measure [1•]. 


\section{Young Versus Old, Type 1 Versus Type 2}

Diabetes mellitus (DM) remains more prevalent in older people compared with younger ones, as are other conditions such as hypertension, renal disease, and the need for joint replacement surgery. Thus, older individuals admitted to hospital are more likely to have diabetes mellitus (DM). Independent of age, glycemic control can become more challenging as a result of stress due to illness or a procedure in patients with or without a diagnose of DM. We can expect to see increasing rates of DM in patients in the Emergency Department (ED) and on inpatient services, due to the ongoing obesity epidemic and the aging population.

DM has traditionally been categorized as type 1 diabetes mellitus (T1DM) and type 2 diabetes mellitus (T2DM), in which type 1 refers to juvenile onset diabetes and type 2 to adult onset, Today, the lines between the two conditions have become blurred. Due to the obesity epidemic, more children, adolescents, and young adults develop type 2 diabetes than in the past. Adults with type 2 diabetes may use basal insulin in addition to oral medications or shorter-acting forms of insulin to achieve glycemic control. Unchanged, however, is the fact that patients with type 1 or juvenile onset diabetes do not produce insulin and those with type 2 , formerly termed adult-onset DM are insensitive to it. These distinctions are important because insulin dosing approaches may differ markedly between patients with type 1 and type 2 diabetes who are in the throes of an acute illness.

\section{Hyperglycemia in the ED}

Given the current prevalence of DM, how best to address hyperglycemia in the ED is an increasingly relevant issue in which the principal goals are to minimize disruption of the metabolic state and achieve a stable glycemic balance as promptly as possible. However, although there is ample evidence in the literature to support attention to blood glucose control in patients who are critically ill or have had an acute MI, the paucity of controlled trials that explore the benefits and risks of "loose" or "tight" glycemic control in other hospitalized patients place emergency physicians and hospitalists in the challenging position of managing patients without a substantial body of evidence to guide them.

\section{Hyperglycemia and Patient Outcomes}

Despite the fact that hyperglycemia is associated with adverse patient outcomes, active intervention to normalize glycemia is associated with inconsistent results. Results of several trials have failed to show significant improvement in mortality with intensive glycemic control [2], or have shown increased mortality risk [3]. Moreover, these and other trials have noted risk of severe hypoglycemia that can result from such efforts [2-7]. These outcomes have contributed to confusion about specific glycemic targets and the means for achieving them in critically and noncritically ill patients.

\section{A Call to Action}

Transition of care from the ED to an inpatient service can result in gaps in comprehensive care, particularly in patients with DM who may have other serious comorbidities. Even in patients without a previous diagnosis of DM, uncontrolled hyperglycemia, regardless of cause during hospitalization, is associated with adverse outcomes and longer lengths of stay $[8,9]$.

The remainder of this paper will focus on a hypothetical diabetic patient who presents to the ED with a non-ST-elevated myocardial infarction (NSTEMI), is found to be hyperglycemic, and will undergo interventional management during hospital admission. His clinical path will follow accepted protocols for NSTEMI, with focus on two continuing possible approaches to the hyperglycemia and management of diabetes: treatment with the traditional sliding scale insulin and treatment with basal insulin. See Table 1 for a case study of hospital-based management of DM.

\section{Inpatient Diabetes Management}

Management of diabetes in hospitalized patients may be directed by primary care physicians, endocrinologists, intensivists, or hospitalists. It has been demonstrated that

Table 1 Case Study

A 62-year-old male patient presents to the ED with sever chest pain at rest. History includes known coronary artery disease (CAD), T2D hyperlipidemia, moderate hypertension, and obesity. His current weight is $100 \mathrm{~kg}$, and his body mass index (BMI) is 35 . He complains of chest pain and nausea; symptoms did not respond to nitroglycerin. He has not eaten sine the previous evening and took no medications today other than aspirin

Current medications include nitroglycerin sublingual as needed, metformin, glyburide, atorvastatin, lisinopril, losartan, hydrochlorothiazide, and aspirin

Laboratory values are unremarkable, with the exception of serum glucose 310 , serum creatinine 2.0 , total cholesterol $280 \mathrm{mg} / \mathrm{dL}$ with low-density lipoprotein at $250 \mathrm{mg} / \mathrm{dL}$ and high-density lipoprotein (HDL) $30 \mathrm{mg} / \mathrm{dL}$; triglycerides $445 \mathrm{mg} / \mathrm{dL}$; initial results of cardiac biomarker assays reveal no sign of myocardial necrosis. ECG shows ST segment depression but is otherwise unremarkable. Chest X-ray is unremarkable

Patient will be admitted to hospital for at least $24 \mathrm{~h}$ of observation and appropriate serial cardiac biomarkers assays 
Table 2 Common barriers to improving and maintaining optimal blood glucose control in hospitalized patients [10]

Fear includes hypoglycemia

Insufficient knowledge of insulin use among house staff

Lack of awareness of the benefits of optimal glycemic control

Complexity of medical conditions and comorbidities

Frequent use of sliding scale insulin regimens

Lack of standardized insulin protocols and order sets

Failure to tailor insulin regimen to individual patient needs

Lack of hypoglycemia protocol

Lack of measures to prevent hypoglycemia

Lack of coordination among blood glucose measurements, meal delivery, and insulin administration

Failure to adjust insulin doses promptly

Changes in nutritional intakes

Exposure to medications that affect blood glucose levels

Transition across different hospital units and care teams

involvement of appropriately trained specialists or specialty teams may reduce length of stay, improve glycemic control, and improve outcomes [9]. This multispecialty approach can be effective only if all members of the medical team have access to a patient's day-to-day treatment notes, laboratory data, and nursing updates. See Table 2 for examples of common barriers to improving and maintaining optimal blood glucose control in hospitalized patients [10].

Electronic medical records (EMRs) can certainly facilitate these goals, and communications protocols are essential. As hospitals begin to comply with "meaningful use" regulations for electronic health records as required under the Health Information Technology Act (HIT), all components of a structured insulin order sets should be incorporated into electronic insulin order sets [11]. A team approach is needed to establish hospital pathways. To achieve glycemic targets associated with improved hospital outcomes, hospitals will need multidisciplinary support to develop insulin management protocols that effectively and safely enable achievement of glycemic targets.

Results of a recent study suggest that systematic attention to hyperglycemia in the emergency department can result in better glycemic control during hospitalization for those patients who are subsequently admitted. The authors of the study conclude that, "Standardization of insulin protocols in the ED and hospital settings leads to improvement in overall glycemic control with greater safety and efficacy than usual care" [12].

\section{Approach to Blood Sugar Management in Patients with Critical and Noncritical Illness}

Hyperglycemia is defined as blood glucose $>140 \mathrm{mg} / \mathrm{dL}$. An $\mathrm{HbA1c}$ value $\geq 6.5 \%$ is diagnostic of diabetes and serves to differentiate preexisting diabetes from stressinduced hyperglycemia. HbA1c is also useful in planning continuity of care, because in patients with diabetes, it provides information about pre-hospital glycemic control and helps to inform discharge planning [8].

A helpful step in considering how to manage hyperglycemia in a hospitalized patient with a noncritical illness is to review the ADA's 2013 standards of care. All patients with diabetes who are admitted to the hospital should have their diabetes clearly documented in the medical record. The next step is to order blood glucose monitoring and to make results available to all members of a patient's health care team $[1 \bullet]$.

\section{Blood Glucose Goals Vary According to Whether a Patient is Critically or Noncritically IIl}

In critically ill patients, insulin therapy should be initiated for treatment of persistent hyperglycemia starting at $\geq$ $180 \mathrm{mg} / \mathrm{dL}(10 \mathrm{mmol} / \mathrm{L})$. After insulin therapy has been started, the standard of care recommends a random glucose range of $140-180 \mathrm{mg} / \mathrm{dL}(7.8-10 \mathrm{mmol} / \mathrm{L})$ for the majority of hospitalized patients. More stringent goals in the range of $110-140 \mathrm{mg} / \mathrm{dL}(6.1-7.8 \mathrm{mmol} / \mathrm{L})$ may be appropriate for some patients, but only if this can be achieved without significant hypoglycemia. Finally, critically ill patients require an intravenous insulin protocol that has demonstrated efficacy and safety in achieving the desired glucose range without increasing risk for severe hypoglycemia [1•]. These goals make a case for the use of basal insulin in hospitalized patients. See Table 3 for examples of blood glucose goals for hospitalized patients.

In noncritically ill inpatients, no clear evidence that supports specific blood glucose goals has come to light, and there are no prospective RCT data to inform glycemic targets in this group of patients. Thus, recommendations must be based on clinical experience and judgment. However, for patients who receive insulin, premeal blood

Table 3 Blood glucose goals for hospitalized patients

Critically ill

Insulin infusion should be used to control hyperglycemia

Starting BG threshold $\leq 180 \mathrm{mg} / \mathrm{dL}(10.0 \mathrm{mmol} / \mathrm{L})$

Glucose level should be maintained between 140 and $180 \mathrm{mg} / \mathrm{dL}$ ( 7.8 and $10.0 \mathrm{mmol} / \mathrm{L}$ )

Lower BG targets may be appropriate for some patients

Noncritically ill

Random blood glucose of $180 \mathrm{mg} / \mathrm{dL}$ (10.0 mmol/L) may be appropriate, but only if such targets can be safely achieved

More stringent targets may be appropriate in stable patients with previously established tight glycemic control 
glucose targets of $140 \mathrm{mg} / \mathrm{dL}(7.8 \mathrm{mmol} / \mathrm{L})$ with random blood glucose of $180 \mathrm{mg} / \mathrm{dL} \quad(10.0 \mathrm{mmol} / \mathrm{L})$ may be appropriate, but only if such targets can be safely achieved. More stringent targets may be appropriate in stable patients with previously established tight glycemic control, and less stringent targets may be appropriate for patients with severe comorbidities $[1 \bullet]$.

The standards of care also state that for noncritically ill patients, scheduled subcutaneous insulin with basal, nutritional (otherwise known as meal time or prandial), and correction components is preferred for achieving and maintaining glucose control. Moreover, glucose monitoring should be standard operating procedure in all nondiabetic patients who receive treatment that carries high risk for hyperglycemia, including high-dose glucocorticoid therapy, enteral or parenteral nutrition, or medications such as octreotide or immunosuppressive agents. If persistent hyperglycemia ( $\geq 180 \mathrm{mg} / \mathrm{dl}$ ) is documented, patients can be treated to glycemic goals that match those for patients with diabetes, and an individualized plan for preventing and treating hypoglycemia should be developed. Episodes of hypoglycemia in the hospital should be documented in the medical record and tracked. For patients with diabetes for whom HbA1c testing results within the past 2-3 months are not available, guidelines recommend that clinicians consider obtaining an $\mathrm{HbA} 1 \mathrm{C}$ on hospital admission. In addition, hospital physicians should also consider obtaining an $\mathrm{HbA} 1 \mathrm{C}$ in patients with risk factors for undiagnosed diabetes who exhibit hyperglycemia while hospitalized [1•].

The ADA recommendations for noncritically ill inpatients conclude with, "Patients with hyperglycemia in the hospital who do not have a prior diagnosis of diabetes should have appropriate plans for follow-up testing and care documented at discharge" [1•].

If we extend these recommendations to patients who present to the ED, then the ADA standard of care strongly supports the use of basal insulin in critically and noncritically ill patients in a hospital setting.

\section{Patient Management: The Case for Basal Insulin}

The traditional model of care among hospital-based physicians has been to treat the diabetic patient with a regular insulin on a sliding scale. It is simple, quick to order, and there is little perceived risk of a patient suffering an episode of hypoglycemia. However, current evidence suggests that while this approach is straightforward, it may not be the best for patient care. A state of persistent hyperglycemia leads to worsened outcomes and an increase of cost of care. A more global management approach to DM is needed. This approach begins and ends with basal and mealtime insulin.

\section{Transitioning Care to the In-Patient Hospital}

Optimal transition of care from the ED to inpatient status requires coordination among ED physicians and hospitalists to ensure continuity of care. The patient's course of care in the ED should be reviewed with the inpatient team. If possible, the rationale for use of specific antihyperglycemic agents, including insulin, should be shared with the receiving team to ensure smooth transition from $\mathrm{ED}$ to hospital service. It is critical that the process begin while the patient is in the ED, as hyperglycemia left untreated while in the ED has a profound impact on the ability of the inpatient team to attain euglycemia.

\section{Basal Insulin Versus Sliding Scale Insulin}

Basal insulin must be differentiated from traditional sliding scale insulin. According to the ADA standards of care, insulin therapy is the preferred method of glycemic control in majority of in-hospital clinical situations. Intravenous infusion is the preferred route of administration in the intensive care unit (ICU). In other hospital settings (including the ED), the standards recommend scheduled subcutaneous insulin that delivers basal, nutritional, and correction (supplemental) components. Typical dosing schemes are based on body weight, with some evidence that patients with renal insufficiency should be treated with lower doses [1•].

\section{Basal Insulin}

Patients who present to the ED or are hospitalized often require an insulin regimen that can change rapidly as their condition changes. A basal/bolus insulin regimen fulfills those criteria and mimics a normal insulin profile [8]. See Fig. 1 for examples of physiologic principles of the basal/ bolus insulin regimen.

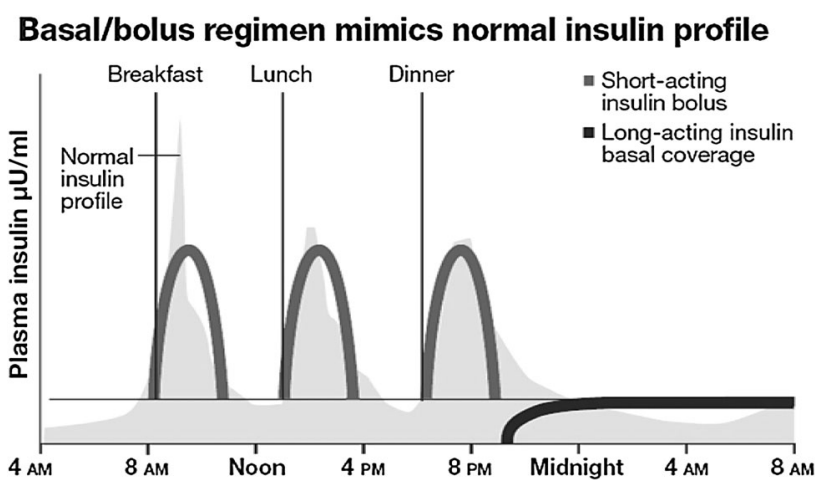

Fig. 1 Physiologic principles of the basal/bolus insulin regimen 
In contrast to a sliding-scale or correction scale insulin regimen, scheduled basal/bolus insulin can prevent hyperglycemia, while sliding or correction scale insulin regimens only lower hyperglycemia after it has occurred. Table 4 shows insulin types, brand names, onset, peak, and duration of action. A comparison of scheduled basal/bolus insulin with sliding scale insulin alone revealed that a significantly higher percentage of patients achieved goal glucose levels in the basal/bolus group compared with those in the sliding scale group (66 vs. $38 \%$ ) without an increase in hypoglycemia [13].

\section{Components of a Basal/Bolus Regimen}

The three components of a basal/bolus regimen are basal insulin, meal or nutritional bolus insulin, and correction insulin (Fig. 1). The ideal basal insulin provides a constant

Table 4 Injectable diabetes medications available in the USA [15••]

\begin{tabular}{|c|c|c|c|c|}
\hline Insulin type & Product & Onset & Peak & Duration \\
\hline \multicolumn{5}{|c|}{ Rapid-acting (bolus) } \\
\hline $\begin{array}{l}\text { Insulin aspart } \\
\text { analog }\end{array}$ & NovoLog & $10-30 \mathrm{~min}$ & $30 \mathrm{~min}-3 \mathrm{~h}$ & $3-5 \mathrm{~h}$ \\
\hline $\begin{array}{l}\text { Insulin } \\
\text { glulsine } \\
\text { analog }\end{array}$ & Apidra & & & \\
\hline $\begin{array}{l}\text { Insulin lispro } \\
\text { analog }\end{array}$ & HumaLog & & & \\
\hline \multicolumn{5}{|c|}{ Short-acting (bolus) } \\
\hline $\begin{array}{l}\text { Human } \\
\text { regular }\end{array}$ & $\begin{array}{l}\text { Humulin R } \\
\text { Novolin R }\end{array}$ & $30-60 \mathrm{~min}$ & $2-5 \mathrm{~h}$ & $\begin{array}{l}\text { Up to } \\
12 \mathrm{~h}^{\mathrm{a}}\end{array}$ \\
\hline \multicolumn{5}{|c|}{ Intermediate-acting (bolus) } \\
\hline $\begin{array}{l}\text { Human NPH } \\
\text { insulin }\end{array}$ & $\begin{array}{l}\text { Humulin N } \\
\text { Novolin N }\end{array}$ & $90 \min -4 \mathrm{~h}$ & $4-12 \mathrm{~h}$ & $\begin{array}{l}\text { Up to } \\
24 \mathrm{~h}^{\mathrm{b}}\end{array}$ \\
\hline \multicolumn{5}{|c|}{ Long-acting (basal) } \\
\hline $\begin{array}{l}\text { Insulin } \\
\text { determir }\end{array}$ & Levemir & $45 \min -4 \mathrm{~h}$ & $\begin{array}{c}\text { Minimal } \\
\text { peak }\end{array}$ & $\begin{array}{l}\text { Up to } \\
24 \mathrm{~h}^{\mathrm{c}}\end{array}$ \\
\hline $\begin{array}{l}\text { Insulin } \\
\text { glargine }\end{array}$ & Lantus & & & \\
\hline
\end{tabular}

Premixed insulin combinations (bolus)

\begin{tabular}{ll}
\hline Insulin type & Product \\
\hline $70 \%$ NPH: $30 \%$ regular & Humulin 70/30 \\
$70 \%$ NPH: $30 \%$ regular & Novolin 70/30 \\
$50 \%$ lispro protamine suspension 50\% lispro & Humalog Mix 50/50 \\
$75 \%$ lispro protamine suspension 25\% lispro & Humalog Mix 75/25 \\
$70 \%$ aspart protamine suspension 30\% aspart & Novolog Mix 70/30
\end{tabular}

\footnotetext{
${ }^{a}$ Usual clinical relevance can be less than $12 \mathrm{~h}$

${ }^{\mathrm{b}}$ Usual clinical relevance can be less than $24 \mathrm{~h}$. Often requires twice daily dosing

${ }^{\mathrm{c}}$ Individual response may require twice daily dosing
}

24-h "peakless," or tonic insulin level to suppress hepatic glucose release during the fasting state and between meals. Glargine and detemir deliver relatively peakless basal insulin. Glargine is preferred because it has a longer duration of action and permits true once-daily administration. When used appropriately, basal insulin should not cause hypoglycemia in patients who are restricted from oral nutritional intake (NPO).

The purpose of mealtime bolus insulin is to prevent predictable postprandial rise in glucose. Thus, bolus insulin can be given at each meal with one of the rapid-acting analogs (lispro, aspart, or glulisine). These insulin analogs offer rapid onset of action and typically reach peak levels within $60 \mathrm{~min}$. Rapid-acting insulin analogs should be administered within $15 \mathrm{~min}$ before a meal, but regular insulin, due to its slower onset of action, must be given at least $30 \mathrm{~min}$ before a meal.

The purpose of correction insulin is to lower hyperglycemic glucose levels, rather than to cover nutritional hyperglycemia. However, in common with mealtime bolus insulin, rapid-acting analog formulations are a good choice for correctional insulin for patients who can eat [8].

\section{Sliding Scale Insulin}

Prolonged therapy with sliding-scale insulin (SSI) as the sole regimen is ineffective in the majority of patients, increases risk of both hypoglycemia and hyperglycemia, and has recently been shown in a randomized trial to be associated with adverse outcomes in general surgery patients with type 2 diabetes [13]. Note that SSI is potentially dangerous in type 1 diabetes [9].

In an inpatient setting, an insulin protocol based on standing orders in which one size does not fit all is typically used. Patients may receive a sliding scale regimen even if their pre-hospital HbA1c was within satisfactory limits. In actual use, the sliding scale typically depends on blood glucose levels that are tested every $6 \mathrm{~h}$ at bedside. In this setting, hospitalized patients may not be treated with basal insulin, and, given rapid-acting insulin acts for only 3-4 h, glucose test results reflect the intake of short-acting (regular) insulin alone, which lasts about 6-8 h, depending on dose. Another disadvantage of a sliding scale regimen is that it tends to have a cutoff point below which no insulin is administered. However, omitting a dose because a patient's glucose level has declined below the cutoff point can mean that no insulin is available for many hours and a consequent spike in blood sugar [14].

\section{NPH Insulin}

NPH insulin should be avoided in hospitalized patients because it is characterized by a pronounced and variable 
peak and is associated with unpredictable hypoglycemia, particularly in patients who are not eating reliably [8].

Table 4 shows rapid-acting, short-acting, intermediateacting, and long-acting (basal) insulins available in the United States according to insulin type, brand name, onset of action, peak, and duration of activity, along with premixed insulin combinations by type and brand names $[15 \bullet \bullet$.

\section{Role of Oral and Noninsulin Parenteral Medications}

Under most circumstances, patients should discontinue use of oral agents during acute illness unless hospitalization is to be very brief [8].

- Metformin must NOT be used in patients who may require use of iodinated contrast studies (CT) or who have renal insufficiency.

- Use of sulfonylureas and metaglinides is associated with unpredictable hypoglycemia in patients who are not eating reliably.

- Use of thiazolidinediones can result in fluid retention, especially if used in combination with insulin.

- Use of parenteral glucagon-like peptide-1 and amylin agonists is associated with nausea, so these agents should be withheld in acutely ill patients.

The drawbacks to oral agents suggest that inpatient hyperglycemia may be best managed with insulin only. Patients can resume taking oral agents as they approach discharge or transfer to a non-acute setting [8].

\section{Determining Insulin Strategies}

Total daily dose (TDD) of insulin can vary according to whether a patient is currently using insulin or is insulin naïve. For patients who are insulin naïve, a TDD of $0.3-0.6 \mathrm{U} / \mathrm{kg}$ of body weight is considered safe. As shown in Table 5, leaner patients and those with renal insufficiency may be started on the lower dose $(0.3-0.4 \mathrm{U} / \mathrm{kg})$, and those who are obsess or are taking glucocorticoids can begin with the higher dose. Under certain circumstances, patients may require more or less insulin than their body weight would imply. Such circumstances include chronic obstructive pulmonary disease (COPD), use of glucocorticoids, cancer, renal insufficiency, and other acute and chronic conditions.

Patients who use basal-bolus insulin therapy take four injections a day, combining basal insulin and prandial insulin before meals. Basal-bolus insulin therapy allows for dosage flexibility and is well suited for patients with varied food intake or irregular meal patterns. An added advantage
Table 5 Determining a TDD for insulin-naïve patients [8]

\begin{tabular}{ll}
\hline TDD estimation & Patient characteristics \\
\hline $0.3 \mathrm{U} / \mathrm{kg}$ body weight & Underweight \\
& Older age \\
& Hemodialysis \\
$0.4 \mathrm{U} / \mathrm{kg}$ body weight & Normal weight \\
$0.5 \mathrm{U} / \mathrm{kg} /$ body weight & Overweight \\
$0.6 \mathrm{U} / \mathrm{kg} /$ body weight & Obese \\
& Insulin resistant \\
& Glucocorticoids \\
\hline
\end{tabular}

to basal-bolus insulin therapy is that a healthcare provider can adjust insulin doses on the basis of monitored blood glucose levels at each meal [10].

Clinical studies in patients with type 1 and type 2 diabetes have consistently demonstrated that in patients who are eating normally, optimal glycemic control can be achieved with subcutaneous insulin when about $50 \%$ of the TDD is given as basal insulin and the other $50 \%$ as bolus insulin [16]. Keep in mind, however, that mealtime bolus doses must be determined on the basis of how much patients are eating [8].

Premeal prandial insulin doses used on an inpatient basis can be $5 \mathrm{U}$ per meal or approximately $7 \%$ of the basal insulin dose, or, even more simply, or $1 \mathrm{U}$ per $15 \mathrm{~g}$ carbohydrate (i.e. 1 carbohydrate exchange) [10].

If premeal glucose is elevated, supplemental doses of rapid-acting insulin, correction doses, can be added to the mealtime dose. If premeal glucose is low, the dose can be decreased. Hospital staff adjust basal and prandial insulins independently to achieve target A1C levels and blood glucose levels [10].

The classic way to think about the basal/bolus insulin dose calculation is to envision a $100 \mathrm{~kg}$ patient who will take $50 \%$ of his or her insulin as basal insulin and the other $50 \%$ as bolus. The formula is $100 \mathrm{~kg} \times 0.5=50 \mathrm{U}$ total daily dose (TDD). One half of the TDD is long-acting basal insulin or $25 \mathrm{U}$, and $25 / 3$ (for three meals a day) of prandial (aka mealtime or bolus) insulin at each meal.

Another calculation for an $80 \mathrm{~kg}$ patient is shown in Table 6.

Intravenously administered insulin (insulin drip) is the treatment of choice for critically ill (ICU) patients who require insulin. When it is appropriate to transfer from IV insulin to subcutaneously administered insulin as a result of clinical improvement, the transition should not lead to deterioration in glycemic control. However, deterioration in control can occur when SSI is substituted for insulin infusions, or when doses of basal or nutritional insulin (or both) are underestimated or overestimated. It is essential to accurately estimate the initial adjustment to maintain 
Table 6 Basal/bolus insulin dose calculation for an $80-\mathrm{kg}$ patient with BMI of $28 \mathrm{~kg} / \mathrm{m}^{2}$ and normal renal function [8]

\begin{tabular}{|c|c|}
\hline \multirow{2}{*}{ Step 1} & TDD calculation \\
\hline & $\mathrm{TDD}=0.5 \mathrm{U} / \mathrm{kg}$ body weight $\times 80=40 \mathrm{U}$ \\
\hline \multirow[t]{2}{*}{ Step 2} & Basal insulin dose calculation \\
\hline & $\begin{array}{l}\text { Basal insulin dose }=50 \% \text { of TDD }=50 \% \text { of } \\
\quad 40 \mathrm{U}=20 \mathrm{U} \text { glargine }\end{array}$ \\
\hline \multirow[t]{2}{*}{ Step 3} & Bolus insulin dose calculation \\
\hline & $\begin{array}{l}\text { Bolus insulin dose per meal }=(50 \% \text { of TDD }) / \\
3=(50 \% \text { of } 40 \mathrm{U}) / 3=20 / 3=6.3 \mathrm{U} \text {, or } \sim 6 \mathrm{U} \text { of } \\
\text { rapid-acting insulin before each meal. If the patient or } \\
\text { nurse estimates that the patient is only eating } 50 \% \text { of } \\
\text { the food on the tray, a reduced dose of } 3 \mathrm{U} \text { should be } \\
\text { ordered instead of the full dose of } 6 \mathrm{U}\end{array}$ \\
\hline \multirow[t]{2}{*}{ Step 4} & Correctional scale estimation \\
\hline & $\begin{array}{l}\text { Assessment of correctional scale insulin is based on } \\
\text { TDD. For a patient with a TDD of } 40 \mathrm{U} \text {, the low } \\
\text { correctional scale should be ordered }\end{array}$ \\
\hline
\end{tabular}

euglycemia and avoid hypoglycemia. If a patient's insulin hourly rate is fairly stable, 24 -h insulin requirements can be calculated by multiplying the amount of insulin required during the previous $6 \mathrm{~h}$ by 4 . Eighty percent of this amount would result in a potentially appropriate TDD [17, 18].

Table 7 shows a four-step sample conversion from IV to basal/bolus insulin, appropriate for patients who have required IV insulin while in an intensive care unit (ICU) and can transition to subcutaneous administration in preparation for transitioning to a medical service.

\section{Premixed Insulin}

Premixed insulins are available as $70 \% \mathrm{NPH} / 30 \%$ regular, $70 \%$ insulin aspart protamine $/ 30 \%$ insulin aspart, $75 \%$ insulin lispro protamine/25\% insulin lispro, or $50 \%$ insulin lispro protamine $/ 50 \%$ insulin lispro, as shown in Table 4 . These mixtures contain postprandial (shorter-acting) and intermediate-release glucose control. These formulations can be administered at the largest meal once daily or at the two largest meals twice daily. Dosage adjustments are based on the pre-dinner glucose level if administered pre-breakfast, and on the fasting blood glucose level if administered pre-dinner [10].

Premixed insulins are somewhat limited in their ability to reach glycemic targets unless given more frequently or in higher doses, which increases the potential for hypoglycemia and weight gain. The American Association of Clinical Endocrinologists (AACE) in its 2011 comprehensive care plan notes that premixed may be appropriate for patients who have difficulty adhering to a drug regimen. However, such preparations lack component dosage flexibility and can increase risk for hypoglycemia compared with basal insulin or basal-bolus regimens, which are more flexible [10].
Table 7 Basal/bolus insulin dose calculation for a patient started on diet who required $2 \mathrm{U} / \mathrm{h}$ of insulin overnight while NPO [8]

\begin{tabular}{ll}
\hline Step 1 & Basal dose calculation \\
& Patient's hourly insulin infusion rate while NPO $=2 \mathrm{U} / \mathrm{h}$ \\
& 24-h basal insulin dose during stress $=24 \times$ hourly \\
& infusion rate $=24 \times 2=48 \mathrm{U}$ \\
& Adjusted basal dose accounting for stress reduction $=2 /$ \\
& $3 \times 24$-h basal rate $=2 / 3 \times 48=32 \mathrm{U}$ of glargine \\
Step 2 & TDD calculation \\
& TDD $=$ dose is $2 \times$ adjusted basal dose $=2 \times 32=64 \mathrm{U}$ \\
Step 3 & Mealtime bolus dose calculation \\
& Patient just started to eat, so $10 \%$ of bolus dose can be \\
& started with each meal $=0.1 \times 32=3 \mathrm{U}$ \\
Step 4 & Correctional scale estimation \\
& A moderate-level correctional scale is most appropriate for \\
& an estimated TDD of $64 \mathrm{U}$
\end{tabular}

Diabetic patients are associated with longer hospital stay and greater perioperative morbidity and mortality, and are also associated with higher health care costs than in the nondiabetic population. Reasons for increased morbidity and mortality are partly the result of higher incidence of comorbid conditions such as coronary heart disease, hypertension, renal insufficiency and adverse effects of hyperglycemia on clinical outcomes [13]. These facts notwithstanding, hyperglycemia is often overlooked, and if identified, may be inadequately addressed due to fear of hypoglycemia.

Umpierrez and coworkers evaluated the use of basalbolus insulin with glargine once daily plus glulisine before meals as a means of managing blood glucose levels in general surgery patients with T2DM. A total of 211 patients were enrolled, 107 in the SSI (regular insulin sliding scale) group and 104 in the basal/bolus insulin group. Table 8 shows clinical characteristics, type of surgery, and blood glucose values during treatment.

Difference between groups in the frequency of the composite outcome, including wound infection, pneumonia, bacteremia, respiratory failure, and acute renal failure, were higher in the SSI group $(24.3 \%)$ than in basal-bolus group (8.6\%; $P=0.003$ ) (Table 9). There were reductions with basal-bolus as compared with SSI in wound infection (2.9 vs. $10.3 \% ; P=0.05)$, pneumonia (0 vs. $2.8 \% ; P=0.247$ ), and acute renal failure (3.8 vs. $10.3 \%$; $P=0.106)$. In addition, the basal-bolus regimen resulted in lower, but not significant, reduction in postsurgical ICU admissions.

The investigators concluded that the basal/bolus regimen was associated with better glycemic control and a lower rate of hospital complications than use of SSI, and that it did not increase the number of severe hypoglycemic events. Results of this study support the superiority of 
Table 8 Patient characteristics: RABBIT 2 [13]

\begin{tabular}{|c|c|c|c|c|}
\hline & All & SSI & Basal-bolus insulin & $P$ value \\
\hline Number of patients & 211 & 107 & 104 & NS \\
\hline Male/female & $107 / 104$ & $53 / 54$ & $54 / 50$ & NS \\
\hline \multicolumn{5}{|c|}{ Diabetic treatment on admission } \\
\hline Diet alone & 17 & 11 & 6 & NS \\
\hline Oral agents & 153 & 80 & 73 & NS \\
\hline Insulin alone & 22 & 11 & 11 & NS \\
\hline Insulin + oral agents & 20 & 11 & 9 & NS \\
\hline \multicolumn{5}{|l|}{ BG values } \\
\hline Admission $(\mathrm{mg} / \mathrm{dL})$ & $190 \pm 92$ & $184 \pm 80$ & $197 \pm 104$ & NS \\
\hline Randomization & $198 \pm 54$ & $194 \pm 56$ & $202 \pm 51$ & NS \\
\hline Presurgery $(\mathrm{mg} / \mathrm{dL})$ & $178 \pm 71$ & $181 \pm 72$ & $174 \pm 70$ & NS \\
\hline Postsurgery (mg/dL) & $198 \pm 53$ & $195 \pm 52$ & $201 \pm 55$ & NS \\
\hline After 2nd day of $\mathrm{Rx}$ & $159 \pm 42$ & $172 \pm 46$ & $145 \pm 32$ & $<0.001$ \\
\hline \multicolumn{5}{|c|}{ BG values after $24-\mathrm{h}$ treatment, $\%$ readings } \\
\hline$<140 \mathrm{mg} / \mathrm{dL}$ & $41.9 \pm 30.9$ & $31.2 \pm 28$ & $52.9 \pm 30.1$ & $<0.001$ \\
\hline $70-140 \mathrm{mg} / \mathrm{dL}$ & $41.6 \pm 30.3$ & $31.7 \pm 28.1$ & $51.8 \pm 29.2$ & $<0.001$ \\
\hline$>180 \mathrm{mg} / \mathrm{dL}$ & $28.1 \pm 30.7$ & $35.3 \pm 33.5$ & $20.5 \pm 25.5$ & $<0.001$ \\
\hline
\end{tabular}

Table 9 Composite hospital complications and outcomes composite hospital complications [13]

\begin{tabular}{lllll}
\hline & All & SSI & Basal-bolus insulin & $P$ value \\
\hline $\begin{array}{l}\text { Number of patients with complications } \\
\text { Mortality }\end{array}$ & 35 & 26 & 9 & 0.003 \\
$\begin{array}{l}\text { Postsurgery ICU admission (\%) } \\
\text { Length of stay (days) }\end{array}$ & 16 & 1 & 19.6 & 12.5 \\
ICU & & & & \\
Hospital & $2.51 \pm 1.90$ & $3.19 \pm 2.14$ & $1.23 \pm 0.60$ & NS \\
\hline
\end{tabular}

basal-bolus insulin regimens in general surgery patients with T2DM over the use of SSI alone.

\section{Patient Transitions}

Patients transitioning from hospital to community require appropriate education and follow-up if they are to maintain glycemic control and achieve glycemic goals. An organized multidisciplinary team may be best qualified deliver transitional and ongoing care for patients with DM. Members of such a team can include a primary care physician, endocrinologist, physician assistant, nurse practitioner, registered nurse, certified diabetes educator (CDE), dietitian, exercise specialist, and mental health care professional [10].

Patient education should be comprehensive, particularly for newly diagnosed patients, and should stress self-management strategies. At diagnosis and throughout their lives, and particularly during health crises, patients should receive information and counseling about therapeutic lifestyle management that includes medical nutrition therapy (with reduction and modification of caloric and fat intake to achieve weight loss in those who are overweight or obese), appropriate physical activity, avoidance of tobacco products, and adequate quantity and quality of sleep [10].

Optimal post-discharge follow-up should occur over the long term with a primary care physician, an endocrinologist, a diabetes educator, and other health care professionals such as an ophthalmologist, a vascular specialist, a cardiologist, and a nephrologist, as appropriate.

The most critical time in all of in-patient management is the transition from one medical setting to the next. The time for medical errors and miscommunication heightens during these transition points. Providers of hospital care should be very cautious in their approach with patients on insulin or other diabetic agents. Often times, patients are unable to completely comprehend their treatment or follow-up plans post-discharge. It is imperative that we relate all information to the next care provider scheduled to care 
for the patient, i.e. the primary care provider (PCP) or endocrinologist. Transition of care management, as it relates to insulin and diabetic agents, carries more importance than for other typical medications, given the risk of complications from these medications. Communication with a patient's family or caregiver is also extremely useful in situations that involve insulin. Finally, follow-up education and re-education will help the patient understand their disease process and transition back to a healthy lifestyle.

\section{Summary}

Given the current prevalence of DM, how best to address hyperglycemia in the ED is an increasingly relevant issue in which the principal goals are to minimize disruption of the metabolic state and achieve a stable glycemic balance as promptly as possible. Transition of care from the ED to an inpatient service can result in gaps in comprehensive care, particularly in patients with DM who may have other serious comorbidities. Even in patients without a previous diagnosis of DM, uncontrolled hyperglycemia, regardless of cause during hospitalization, is associated with adverse outcomes and longer lengths of stay.

In contrast to a sliding scale or correction scale insulin regimen, scheduled basal/bolus insulin can prevent hyperglycemia, while sliding or correction scale insulin regimens only lower hyperglycemia after it has occurred. The three components of a basal/bolus regimen are basal insulin, meal or nutritional bolus insulin, and correction insulin. The ideal basal insulin provides a constant 24-h tonic insulin level to suppress hepatic glucose release during the fasting state and between meals. The purpose of mealtime bolus insulin is to prevent predictable postprandial rise in glucose. Thus, bolus insulin can be given at each meal with one of the rapid-acting analogs.

Intravenously administered insulin (insulin drip) is the treatment of choice for critically ill patients who require insulin. When it is appropriate to transfer from IV insulin to subcutaneously administered insulin as a result of clinical improvement, the transition should not lead to deterioration in glycemic control. Patients transitioning from hospital to community require appropriate education and follow-up if they are to maintain glycemic control and achieve glycemic goals. Patient education should be comprehensive, particularly for newly diagnosed patients, and should stress self-management strategies.

The most critical time in all of in-patient management is the transition from one medical setting to the next. Transition of care management, as it relates to insulin and diabetic agents, carries more importance than for other typical medications, given the risk of complications from these medications. The ideal management plan for patients with diabetes involves coordination, knowledge-sharing and constant vigilance to ensure that patients remain euglycemic through the entire process as they move from the ED to the hospital and back home again.

Acknowledgments This paper was supported in part by grants from Sanofi and Astra-Zeneca to Hospital Quality Foundation to support the writing group's efforts, and grants and financial support for travel by The Hospital Quality Foundation to Gregory Volturo.

\section{Compliance with Ethics Guidelines}

Conflict of Interest This paper was underwritten in part by a grant from Sanofi to the Hospital Quality Foundation to support the writing group's efforts, including travel. All authors report receiving consulting fees from Sanofi.

Human and Animal Rights and Informed Consent This article does not contain any studies with human or animal subjects performed by any of the authors writing group's efforts, including travel. All authors report receiving consulting fees from Sanofi.

\section{References}

Papers of particular interest, published recently, have been highlighted as:

- Of importance

•• Of major importance

1. American Diabetes Association. Standards of medical care in diabetes 2013. Diabetes Care. 2013;36(Suppl 1):S11-66. Comprehensive summary of current critical issues.

2. Wiener RS, Wiener DC, Larson RJ. Benefits and risks of tight glucose control in critically ill adults: a meta-analysis. JAMA. 2008;300(8):993-1044.

3. Finfer S, Chittock DR, Su SY, et al. Intensive versus conventional glucose control in critically ill patients. $N$ Engl J Med. 2009;360:1283-97.

4. Brunkhorst FM, Engel C, Bloos F, et al. Intensive insulin therapy and pentastarch resuscitation in severe sepsis. $\mathrm{N}$ Engl $\mathrm{J}$ Med. 2008;358:125-39.

5. Krinsley JS, Grover A. Severe hypoglycemia in critically ill patients: risk factors and outcomes. Crit Care Med. 2007;35:2262-7.

6. Van den Berghe G, Wilmer A, Hermans G, et al. Intensive insulin therapy in the medical ICU. N Engl J Med. 2006;354:449-61.

7. Griesdale DE, de Souza RJ, van Dam RM, et al. Intensive insulin therapy and mortality among critically ill patients: a meta-analysis including NICE-SUGAR study data. CMAJ. 2009;180(8):821-7.

8. Magaji V, Johnston JM. Inpatient management of hyperglycemia and diabetes. Clin Diabetes. 2011;29(1):3-9.

9. Moghissi ES, Korytkowski MT, DiNardo M, et al. American Association of Clinical Endocrinologists and American Diabetes Association consensus statement on inpatient glycemic control. Endocr Pract. 2009;15(4):1-17.

10. Pichardo-Lowden AR, Fan CY, Gabbay RA, et al. Management of hyperglycemia in the non-intensive care patient: featuring subcutaneous insulin protocols. Endocr Pract. 2011;17:249-60.

11. Wexler DJ, Shrader P, Burns SM, et al. Effectiveness of a computerized insulin order template in general medical inpatients 
with type 2 diabetes: a cluster randomized trial. Endocr Pract. 2010;33:2181-3.

12. Bernard JB, Munoz C, Harper J, et al. Treatment of inpatient hyperglycemia beginning in the emergency department: a randomized trial using insulins aspart and detemir compared with usual care. J Hosp Med. 2011;6:279-84.

13. Umpierrez GE, Smiley D, Zisman A, et al. Randomized study of basal-bolus insulin therapy in the inpatient management of patients with type 2 diabetes (RABBIT 2 trial). Diabetes Care. 2007;30(9):2181-6.

14. Guthrie DW, Guthrie RA, Hinnen D, et al. It's time to abandon the sliding scale. J Fam Pract. 2011;60(5):266-70.

15. • Joslin Diabetes Center \& Joslin Clinic. Clinical guideline for pharmacological management of type 2 diabetes. 2011. Excellent summary of current evidenced based insulin therapy regimens.
16. Maynard G, Lee J, Phillips G, et al. Improved inpatient use of basal insulin, reduced hypoglycemia, and improved glycemic control: effect of structured subcutaneous insulin orders and an insulin management algorithm. J Hosp Med. 2009;4:3-15.

17. Furnary AP, Braithwaite SS. Effects of outcome on in-hospital transition from intravenous insulin infusion to subcutaneous therapy. Am J Cardiol. 2006;98:557-64.

18. Schmeltz LR, DeSantis AJ, Schmidt K, et al. Conversion of intravenous insulin infusions to subcutaneously administered insulin glargine in patients with hyperglycemia. Endocr Pract. 2006; $12: 641-50$. 